Editorial

\title{
Acknowledgement to Reviewers of Fluids in 2017
}

\section{Fluids Editorial Office}

MDPI AG, St. Alban-Anlage 66, 4052 Basel, Switzerland

Published: 19 January 2018

Peer review is an essential part in the publication process, ensuring that Fluids maintains high quality standards for its published papers. In 2017, a total of 69 papers were published in the journal. Thanks to the cooperation of our reviewers, the median time to first decision was 24 days and the median time to publication was 50 days. The editors would like to express their sincere gratitude to the following reviewers for their time and dedication in 2017:

Abali, B. Emek

Abramov, Rafail

Afkhami, Shahriar

Alain, Liné

Anderson, Gary A.

Anselmet, Fabien

Arastoopour, Hamid

Baek, Jehyun

Balk, Alexander M.

Bardos, Claude

Barletta, Antonio

Basha, Omar

Bedeaux, Dick

Bellos, Vasilis

Berselli, Luigi

Besagni, Giorgio

Birken, Philipp

Bisi, Marzia

Blacker, Thomas

Boczek, Tomasz

Boreyko, Jonathan

Brujic, Jasna

Buschmann, Matthias $\mathrm{H}$.

Butler, Jason E.

Cai, Mingchao

Carmi, Shlomo

Carton, Xavier

Castro, Ian

Celli, Michele

Chan, Leon

Chaudhry, Hassam

Chemke, Rei

Chen, $\mathrm{Ru}$

Chesneau, Xavier

Chidyagwai, Prince

Chiogna, Gabriele
Chrigui, Mouldi

Chung, Bong Jae

Clegg, Paul

Conti, Michele

Costabile, Pierfranco

Croce, Giulio

D'Onofrio, Alberto

Dayal, Kaushik

De Nijs, Bart De Nijs

Deremble, Bruno

Doostmohammadi, Amin

Dritselis, Chris

Dykas, Sławomir

Ebara, M.

Ellahi, Rehmat

Escola, José María

Esmaily-Moghadam, Mahdi

Espino, Daniel M.

Falsaperla, Paolo

Flack, Karen A.

Foucaut, Jean-Marc

Fusi, Lorenzo

Gallo, Diego

Galtier, Sébastien

Gamez-Montero, Pedro Javier

Garaud, Pascale

Garnier, Bertrand

Ginelli, Francesco

Girton, James

Goubergrits, Leonid

Griffiths, Ian

Griffiths, Ross

Grooms, Ian

$\mathrm{Gu}$, Yibei

Hamed, Ali M.

Hansen, Alex 
Harlander, Uwe

Hassanzadeh, Hassan

Haynes, Peter

Henry, Daniel

Hewitt, Duncan Robin

Higgins, Brian

Hill, Peter Alec

Hinrichsen, Olaf

Hirata, Silvia

Houim, Ryan W.

Huang, Hsin-Fu

Huang, Weimin

Iervolino, Michele

Ikeo, Shigeru

Iliescu, Traian

Irannejad, Abolfazl Mani

Isaac, Kakkattukuzhy M.

Ito, Kazuhide

Iungo, Giacomo

Jaeger, Heinrich M.

Jenko, Frank

Karimipour, Arash

Kim, Eun-Jin

Kim, Hyoungsoo

Kim, Min Chan

Kishen, Anil

Koshel, Konstantin

Kovacic, Gregor

Kuang, Shibo

Kuester, Matthew

Labovsky, Alexander E.

Langella, Ivan

Lapeyre, Guillaume

Larcher, Thomas Von

Laurien, Eckart

Le Clainche, Soledad

Leclercq, Loïc

Li, Gaojin

Li, Xiaolin

Liu, Don

Liu, Minglu

Liu, Yonggang

López-Jiménez, Petra Amparo

Makhaldiani, Nugzar

Malvé, Mauro

Mamou, Mahmoud

Marinkovic, Dragan

Martel-Foley, Joseph

Martys, Nicos

Mathijssen, Arnold J. T. M.

McIntyre, M.E.

McWilliams, James C.
Mei, Renwei

Minev, Peter D.

Mohan, Ram V.

Morrison, P.J.

Muller, Annette

Nadiga, Balasubramanya T.

Nazarenko, Sergey

Neda, Monika

Nguyen, Quoc

Nield, Don

Nolan, David S.

O'Hare, Anthony

Onoue, Kyohei

Örlü, Ramis

Ouarzazi, Mohamed Najib

Pakzad, Leila Pakzad

Pal, Rajinder

Papavassiliou, Dimitrios V.

Parker, Jeff

Pham, Ngoc

Pinelli, Alfredo

Poncet, Sébastien

Pop, I

Poulin, Francis

Radko, Timour

Ranade, Vivek V.

Rashidi, Mohmad Mehdi

Rayz, Vitaliy

Rebholz, Leo

Rees, D. Andrew

Reinaud, Jean Noel

Rionero, Salvatore

Robins, Alan

Rotunno, Richard

Ryan, Shawn D.

Ryu, Sungmin

Sader, John

Sagaut, Pierre

San, Omer

Schilling, Oleg

Scholle, Markus

Schoonover, Joseph

Schröder, Andreas

Seo, Jung-Hee

Sequeira, Adélia

Shadloo, Mostafa Safdari

Shafahi, Maryam

Shapiro, Benjamin

Shevchenko, Igor

Siddheshwar, Pradeep

Siddiqui, Javed

Singh, Rajesh 
Sivasubramanian, Jayahar

Smith, Shafer

Spagnolie, Saverio E

Spall, Michael A.

Stern, Daniel

Sullivan, Pierre E.

Sun, Liang

Sutyrin, Georgi G.

Swaters, Gordon E

Sydora, Richard.D.

Taler, Dawid

Tanaka, Yuki

Teixeira, Andrew R.

Tilton, Nils

Torres-Diaz, Isaac

Torrilhon, Manuel

Tran, Minh-Binh

Tran, P.X.

Trevelyan, Philip

Udaykumar, H.S.

Vadasz, Peter

Van Oijen, Jeroen A.
Vergara, Christian

Vinuesa, Ricardo

Vowinckel, Bernhard

Wang, Hang

Wang, Zuankai

Watanabe, Takashi

Wen, Baole

Wen, Chuang

Wessel-Berg, Dag

Wick, Thomas

Wilson, Helen J.

$\mathrm{Wu}$, Huixuan

$\mathrm{Wu}$, Wei-Tao

$\mathrm{Xi}, \mathrm{Li}$

Yadav, Dhananjay

Yang, Dae Ryook

Yoo, Do Guen

$\mathrm{Yu}$, Simon

Zaccone, Alessio

Zamansky, Remi

Zhou, Kai

(C) 2018 by the authors. Licensee MDPI, Basel, Switzerland. This article is an open access article distributed under the terms and conditions of the Creative Commons Attribution (CC BY) license (http://creativecommons.org/licenses/by/4.0/). 